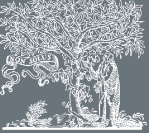

ELSEVIER

\title{
Field distribution and activity of chlorinated solvents degrading bacteria by combining CARD-FISH and real time PCR
}

\section{B. Matturro ${ }^{1}$, F. Aulenta ${ }^{1}$, M. Majone ${ }^{2}$, M. Petrangeli Papini ${ }^{2}$, V. Tandoi ${ }^{1}$ and S. Rossetti ${ }^{1}$,}

${ }^{1}$ Water Research Institute, CNR, Via Salaria km 29.300, Monterotondo (RM) 00015, Italy

${ }^{2}$ Dept. of Chemistry, Sapienza University of Rome, P.le A. Moro 5, 00185 Rome, Italy

Nowadays several advanced molecular techniques are applied for quantifying bacteria involved in contaminant degradation processes. However, despite the fact that significant efforts have been taken to make these tools more reliable and specific, their application for the analysis of field samples is hardly ever applied.

In this study, a combination of three methods (CARD-FISH, qPCR and RT-qPCR) was successfully applied to evaluate the distribution and the activity of known chlorinated solvent dechlorinating bacteria in a contaminated site where no remedial actions have been undertaken.

CAtalysed Reporter Deposition Fluorescence In Situ Hybridization (CARD-FISH) specifically provided the cell densities of known dechlorinating bacteria and was found to be more sensitive than quantitative PCR (qPCR) for the quantification of 'Dehalococcoides' cell numbers in the aquifer. Among the screened dechlorinators, 'Dehalococcoides' spp. were mainly found and nearly homogenously distributed in the aquifers at concentrations ranging from $8.1 \times 10^{5} \pm 1.2 \times 10^{5}$ to $2.5 \times 10^{7} \pm 5.6 \times 10^{6}$ cells per liter of groundwater (with a relative abundance out of the total Bacteria of 0.7-15\%). Further, the dechlorination potentialities of 'Dehalococcoides' species living in the aquifer were evaluated by analyzing the abundance and the expression of 16S rRNA genes and reductive dehalogenase (RDase) encoding functional genes by qPCR and Reverse Transcription qPCR (RT-qPCR). 'Dehalococcoides' tceA gene, known to be associated to strains capable of reducing chlorinated solvents beyond cis-DCE, was found and expressed in the field. Overall, this study proved the existence of a well-established dechlorinating microbial community able to use contaminants as substrates for their metabolic activity and indicated the occurrence of reductive dechlorination at the site.

\section{Introduction}

Bioremediation offers a promising method for the reduction and elimination of man-made compounds in the environment. Persistent pollutants such as chlorinated ethenes (i.e. perchloroethylene, PCE and trichloroethylene, TCE) are commonly found in the environment due to their widespread use in a variety of commercial and industrial applications. Because chlorinated solvents are highly toxic and mobile in groundwater, they represent a serious risk for human health and the environment.

These compounds can be dechlorinated under anaerobic conditions by halorespiring bacteria through a well-known process named reductive dechlorination (RD). Although some microbial species are known to be involved in the partial degradation of PCE or TCE to cis-dichloroethylene (cis-DCE) (i.e. Sulfurospirillum spp., Desulfuromonas spp., Desulfitobacterium spp., Dehalobacter spp. and Geobacter spp.) [1], till now only members of 'Dehalococcoides' 
genus are able to completely dechlorinate such compounds to harmless ethene [2-5].

$\mathrm{RD}$ led by 'Dehalococcoides' spp. is mediated by key-degradative enzymes (reductive dehalogenase, RDase), whose strain specificity was already proved in several studies $[2-4,6,7]$. Three main functional genes, tceA, $b v c A$ and $v c r A$, involved in different steps of the reductive dechlorination process, are used as biomarkers to assess the activity of 'Dehalococcoides' strains. In detail, these genes code for proteins catalysing the metabolic dechlorination of TCE to cisDCE and vinyl chloride (VC) and for the cometabolic production of ethene (tceA gene, in strains 195 and FL2), in the dechlorination step from VC to ethene ( $b v c A$, in strain BAV1) and in the reduction of DCE and VC to ethene (vcrA, in strains VS and GT) [4,5,8-13]. This peculiarity allows the use of 'Dehalococcoides' genus as biomarker of chlorinated solvents contamination and its detectability in the field can reveal the occurrence of on-going biodegradative processes. Much attention should be, therefore, paid on the use of rapid and reliable screening tools allowing the biomonitoring of key-degradative bacteria in site samples.

Because cultivation techniques are laborious and not always applicable, a variety of molecular techniques have been developed, primarily based on PCR, to study these microorganisms in mixed cultures [14]. This approach allows to specifically quantify 'Dehalococcoides' spp. 16S rRNA genes and to discriminate among strains with different metabolic peculiarities by targeting key-functional genes. In particular, quantitative PCR protocols were developed for $t c e A, v c r A$ and $b v c A$ genes involved in the reductive dechlorination process and were successfully applied to quantify these genes and their expression level in pure cultures, lab-scale microbial enrichments $[15,16]$ and, to a minor extent, at biostimulated or bioaugmented aquifers [17-21].

qPCR has contributed greatly to our understanding of 'Dehalococcoides' spp. and still represents the method mostly used to quantify microorganisms in environmental samples. However, its application for the enumeration of key-degrading bacteria at field scale may be affected by some known methodological limitations which hinder the sensitive and reproducible application of this approach on environmental samples. Limitations of the technique are mainly linked to DNA/RNA extraction methods employed, the accuracy and the standardization of gene copy number determined by PCR inhibition and/or bias in amplification [1].

In this context, the biomonitoring of key-degrading bacteria in site samples would be improved by the combined use of additional methodologies, independent from nucleic acid extraction and amplification. Fluorescence In Situ Hybridization (FISH) and its variant CAtalysed Reporter Deposition FISH (CARD-FISH) enabled the microscopic enumeration of whole cells of 'Dehalococcoides' spp. [22-25]. This methodology is independent of DNA extraction and it is characterized by detection limits lower than those commonly reported for qPCR [26]. Additionally, the typical pyramidal characterization by FISH probes with different phylogenetic specificity, from large (i.e. domain) to smaller microbial groups (i.e. phylum, genus), allows to estimate the identified fraction out of the whole microbial community. This advantage is not negligible because it provides the confirmatory evidence, always recommendable when complex samples are analyzed, about the level of knowledge acquired after the analysis.
Despite the fact that in the past years in situ fluorescence hybridization technique has gained widespread acceptance in the scientific community as a powerful tool for the screening of microbial populations and their dynamics in environmental samples [27], only few applications on dechlorinating mixed cultures are reported and still fewer on groundwater contaminated by chlorinated solvents $[17,22-24,28-31]$. Here we report the abundance, distribution and activity of chlorinated solvents degrading bacteria in groundwater samples ascertained by the simultaneous application of CARD-FISH, qPCR and RT-qPCR. The combined application of such rapid, reliable and specific biomonitoring tools allowed to estimate cell densities of dechlorinating bacteria with different metabolic attitudes for chlorinated solvents (i.e. 'Dehalococcoides' spp. and Dehalobacter spp.) and to estimate the abundance and gene expression of $16 \mathrm{~S}$ rRNA and tceA, $b v c A, v c r A$ reductive dehalogenase (RDase) encoding genes for evaluating the activity of 'Dehalococcoides' strains at field scale.

\section{Material and methods}

\section{Site description and groundwater sampling}

Samples were collected from an aquifer historically contaminated by chlorinated solvents, located within an industrial area in Northern Italy (Rho, Milano). Groundwater samples from 13 monitoring wells were collected after purging each well for about $30 \mathrm{~min}$. Samples were differently treated for PCR-based and in situ - hybridization applications. For DNA and RNA extractions, 4-5 L of groundwater were filtered respectively, through $0.2 \mu \mathrm{m}$ surfactant-free cellulose ( $\varnothing 47 \mathrm{~mm}$, Millipore) and $0.2 \mu \mathrm{m}$ PVDF filters (Ø142 mm, Millipore). Each filter was rolled with sterile forceps, placed carefully into a $50 \mathrm{~mL}$ plastic sterile tube and transported in dry ice until they arrive in the laboratory for processing within 24 hours as described below.

For CARD-FISH analysis, $45 \mathrm{~mL}$ of water samples were immediately fixed with formaldehyde ( $2 \%, \mathrm{vol} / \mathrm{vol}$ final concentration), filtered through $0.2 \mu \mathrm{m}$ polycarbonate filters ( $647 \mathrm{~mm}$, Millipore) by gentle vacuum $(<0.2 \mathrm{bar})$ and filters were stored at $-20^{\circ} \mathrm{C}$ for successive analysis. At least two different filters were prepared for each groundwater sample.

\section{Chemical and geochemical analyses}

Groundwater samples to be analyzed for volatile hydrocarbons and anions were collected in $20 \mathrm{~mL}$ borosilicate vials, capped with Teflon-lined rubber septa. Chlorinated solvents (i.e. chloroethanes and chloroethenes), methane, ethane and ethene were analyzed in the headspace of the vials by gas-chromatography (GC) with flame ionization detector (FID), using a method described previously [32]. Anions (nitrate, nitrite, sulfate, chloride) were analyzed by ion-chromatography [32]. Total Fe, Fe(II), Fe(III) and COD were determined (on site) using spectrophotometric field sampling kits (Spectroquant, Merck, DE). Dissolved oxygen, temperature, redox potential, $\mathrm{pH}$ and conductivity were measured in situ, by placing a multiparametric probe (Aquamaster Mod. 345, AMEL) directly within the monitoring well.

\section{CARD-FISH}

CARD-FISH assays were performed on filter harvested cells to quantify Bacteria and Archaea domain (Eub338mix and Arch917 
probes) and 'Dehalococcoides' genus (Dhe1259c and Dhe1259t probes) [25] according to previously published procedures $[4,33]$. Other dechlorinating microorganisms which were able to grow on highly chlorinated compounds, such as Geobacter spp. (GEO432 probe), Dehalobacter spp. (DHB643 probe), Desulfuromonas spp. (DRM432 probe), Sulfurospirillum spp. (SULF220ab probe) and Desulfitobacterium spp. (DSF440 probe and DSF475helper probes), were also investigated. GNSB941 probe specific for Chloroflexi phylum [23] was also employed for the analysis of filtered groundwater samples. The probes, labeled at $5^{\prime}$ end with horseradish peroxidase (HRP), were purchased from BIOMERS (Germany). Details of the oligonucleotide probes used in this study are available in Rossetti et al., 2008 or at http://www.microbialecology.net/probebase/ [34]. DAPI staining was always performed for the quantification of the total cells. At least ten different fields of view were selected randomly for each reported population measurement. Error bars are calculated as standard deviations of cell counting performed on at least two different filter sections. Slides were examined by epifluorescence microscopy (Olympus, BX51) and the images were captured with Olympus XM10 camera and handled with Cell-F software (Olympus, Germany).

\section{$D N A$ extraction and quantitative real time $P C R$ ( $q P C R$ )}

Filtered groundwater (4-5 L) was treated by adding $10 \mathrm{~mL}$ of phosphate buffered saline (PBS) $1 \times$ into a sterile $50 \mathrm{~mL}$ tube and vortexing the filters for about $5 \mathrm{~min}$ to dislodge and suspend the cells. The suspended biomass was centrifuged at $14,000 \times g$ for 15 min and the pellet used for DNA extraction. Four DNA extraction procedures were tested on 10 different groundwater samples taken from Rho site. The procedures included Ultra Clean Water DNA Isolation Kit (MoBio - Carlsbad, CA), PowerSoil DNA Kit (MoBio - Carlsbad, CA), Ultra Clean Microbial DNA Isolation Kit (MoBio - Carlsbad, CA) and a phenol:chloroform:isoamyl alcohol protocol [35]. The latter procedure yielded from 1.8 up to 9.4 fold more DNA concentration than the other tested extraction protocols and was therefore chosen for the analysis of groundwater samples. Genomic DNA was eluted in $100 \mu \mathrm{L}$ TE buffer, quantified by spectrophotometer Nanodrop 3300 (Thermo Scientific, Italy) and stored at $-20^{\circ} \mathrm{C}$.

Real-time PCR was performed with absolute quantification method using TaqMan ${ }^{\mathbb{R}}$ probe chemistry with 6-carboxyfluoresceine (FAM) as $5^{\prime}$ end reporter fluorophore and $\mathrm{N}, \mathrm{N}, \mathrm{N}^{\prime}, \mathrm{N}^{\prime}$-tetramethyl-6-carboxyrhodamine (TAMRA) as $3^{\prime}$ end quencher purchased from Applied Biosystems (Italy). Primers and probe sets used to quantify Bacteria 16S rRNA gene and 'Dehalococcoides' spp. 16S rRNA, tce $A, b v c A$ and $v c r A$ genes were previously designed and validated by Ritalahti et al., 2006 [36] and purchased from Eurofins MWG Operon (Germany). The reactions were conducted in triplicate in a total volume of $25 \mu \mathrm{L}$. Each reaction contained TaqMan $^{\circledR}$ Universal PCR Master Mix $2 \times$ (Applied Biosystems, Italy), $300 \mathrm{~nm}$ concentration of each probe and primers and $3 \mu \mathrm{L}$ of 1:10 and 1:100 diluted genomic DNA. qPCR runs included three not template controls for each targeted gene to control contamination events eventually developed during the preparation of the reactions. Reactions were carried out for $2 \mathrm{~min}$ at $50^{\circ} \mathrm{C}$ to activate the AmpErase uracil N-glycosylase, followed by $10 \mathrm{~min}$ denaturation step at $94^{\circ} \mathrm{C}$ for $10 \mathrm{~min}$ and 40 cycles at $95^{\circ} \mathrm{C}$ for $15 \mathrm{~s}$ and $60^{\circ} \mathrm{C}$ for $1 \mathrm{~min}$. Reactions were conducted in 96 wells plates and quantitative data were analyzed using thermocycler Applied Biosystems 7300 System Sequence Detection - Software version 1.3 (SDS v.1). Fluorescence signal data were collected at the final step of each amplification stage $\left(60^{\circ} \mathrm{C}\right.$ for $\left.1 \mathrm{~min}\right)$. The absolute quantification of the targeted genes was performed by interpolating $C_{\mathrm{T}}$ values of unknown samples from the standard curve, the latter prepared with serial dilution of known quantity of long amplicons of gene copies for each targeted gene (i.e. 16S rRNA, tceA, bvcA, vcrA). Details on standards preparation and qPCR efficiencies of the calibration curves are reported in Supporting Information (Figure S1, Table S1).

RNA extraction and quantitative reverse transcription $P C R$ (RTqP(R)

All benches and plastics used for RNA extraction were treated with RNaseZAP $^{\circledR}$ (Sigma, USA) to limit the problems with RNA degradation. Filters with biomass collected from 4 to $5 \mathrm{~L}$ of groundwater sample, were treated by adding $5 \mathrm{~mL}$ of Gibco ${ }^{\circledR}$ distilled DNase/ RNase free water (Qiagen, USA) and 1 volume of RNA protect Bacteria Reagent (Qiagen, USA) into a sterile tube and vortexed briefly for $5 \mathrm{~min}$ at room temperature to dislodge the cells. The suspended biomass was centrifuged at $18,000 \times g$ for $5 \mathrm{~min}$ at $4{ }^{\circ} \mathrm{C}$, the filter and the supernatant were removed and the pellet was stored at $-80^{\circ} \mathrm{C}$. RNA was extracted according to Johnson et al., 2005 [7] protocol and $8.5 \times 10^{7}$ copies of the internal reference luciferase mRNA (Promega, Italy) were added to the pellet during the lysis step. The use of the internal reference served to determine the loss of RNA during all the experimental procedures before the RT-qPCR quantification through the estimation of the percentage of luciferase mRNA recovery in each sample (calculated as difference between the initial copy numbers added during the lysis step and the final copy numbers detected by RT-qPCR).

Extracted RNA was treated to remove contaminating DNA by enzymatic reaction with 4 U DNase I using TURBO DNA-free ${ }^{\mathrm{TM}}$ Kit (Ambion) in a $50 \mu \mathrm{L}$ final volume. Purified RNA was quantified by NanoDrop 3300 (Thermo Scientific, Italy). Aliquots were stored at $-80^{\circ} \mathrm{C}$ and used for one-step RT-qPCR absolute quantification, which included the reverse transcription of RNA and the PCR amplification in a single tube preparation. Gene expression quantitative assays were performed by using the same primers and probe sets employed for the qPCR absolute quantification of the gene copy numbers. Furthermore, in addition to $16 \mathrm{~S}$ rRNA, tceA, bvcA and vcrA primers and probe sets used in qPCR, a set of primers and probe previously designed and validated [7] for the quantification of the luciferase mRNA was employed.

RT-qPCR reactions were performed in triplicate for each unknown sample in a total volume of $25 \mu \mathrm{L}$ with TaqMan $^{\mathbb{R}}$ One Step RT-PCR Master Mix Reagents Kit (Applied Biosystems, Italy) which contains MultiScribe ${ }^{\mathrm{TM}}$ Reverse Transcriptase to synthesize a complementary DNA (cDNA) strand, 300 nм concentration of each probe and primers and $3 \mu \mathrm{L}$ of diluted RNA. Thermal cycling parameters adopted for one step RT-qPCR included the reverse transcription stage at $48^{\circ} \mathrm{C}$ for $30 \mathrm{~min}$, followed by $94^{\circ} \mathrm{C}$ for $10 \mathrm{~min}$ and 40 cycles of amplification at $95^{\circ} \mathrm{C}$ for $15 \mathrm{~s}$ and $60^{\circ} \mathrm{C}$ for $1 \mathrm{~min}$.

As reported for qPCR, reactions were conducted in 96 well plates and quantitative data analyzed with Applied Biosystems 7300 System Sequence Detection - Software version 1.3 (SDS v.1), by 
TABLE 1

\begin{tabular}{|c|c|c|c|c|c|c|c|c|}
\hline Wells & $\begin{array}{l}1,1,2,2-\mathrm{TeCA} \\
\left(\mu \mathrm{g} \mathrm{L}^{-1}\right)\end{array}$ & $\begin{array}{l}\text { PCE } \\
\left(\mu \mathrm{g} \mathrm{L}^{-1}\right)\end{array}$ & $\begin{array}{l}1,1,2-\mathrm{TCA} \\
\left(\mu \mathrm{g} \mathrm{L}^{-1}\right)\end{array}$ & $\begin{array}{l}\text { TCE } \\
\left(\mu \mathrm{g} \mathrm{L}^{-1}\right)\end{array}$ & $\begin{array}{l}\text { cis-DCE } \\
\left(\mu \mathrm{g} \mathrm{L}^{-1}\right)\end{array}$ & $\begin{array}{l}\text { Total chlorinated } \\
\text { solvents }\left(\mu \mathrm{g} \mathrm{L}^{-1}\right)^{\mathrm{a}, \mathrm{b}}\end{array}$ & $\begin{array}{l}\text { Nitrate } \\
\left(\mathrm{mg} \mathrm{L}^{-1}\right)\end{array}$ & $\begin{array}{l}\text { Sulfate } \\
\left(\mathrm{mg} \mathrm{L}^{-1}\right)\end{array}$ \\
\hline PE (A0) & 1177 & 55 & 38 & 365 & 0 & 1634 & 26 & 265 \\
\hline 345 (A0) & 281 & 75 & 24 & 68 & 0 & 447 & 34 & 39 \\
\hline 347 (A0) & 127 & 6 & 0 & 405 & 13 & 538 & 25 & 119 \\
\hline 346 (A1) & 2473 & 14 & 0 & 30,052 & 7 & 32,552 & 16 & 48 \\
\hline 348 (A1) & 162 & 50 & 59 & 4370 & 0 & 4649 & 26 & 122 \\
\hline 597 (A1) & 517 & 29 & 0 & 135 & 0 & 689 & 24 & 39 \\
\hline 601 (A1) & 792 & 13 & 0 & 32 & 16 & 837 & 26 & 37 \\
\hline 663 (A1) & 5981 & 262 & 115 & 11,791 & 0 & 18,166 & 24 & 39 \\
\hline 666 (A1) & 332 & 7 & 0 & 44 & 18 & 383 & 27 & 147 \\
\hline 669 (A1) & 286 & 101 & 262 & 8983 & 0 & 9650 & 28 & 144 \\
\hline 670 (A1) & 363 & 15 & 40 & 362 & 0 & 780 & 22 & 37 \\
\hline 790 (A1) & 445 & 62 & 134 & 6550 & 0 & 7192 & 30 & 86 \\
\hline 349 (A1) & 729 & 17 & 0 & 50 & 0 & 796 & 36 & 49 \\
\hline
\end{tabular}

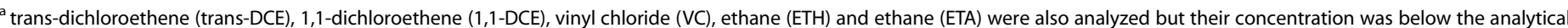
detection limit of the GC-FID.

${ }^{\mathrm{b}}$ Standard deviation of replicated samples was typically below $15 \%$.

interpolating $C_{\mathrm{T}}$ values of unknown samples from the standard curve (Figure S1) of each targeted gene. Serial dilutions of known quantity of long amplicons for each targeted gene were prepared to assess the standard curves (see Supplementary Material for details, Figure S1).

\section{Statistic analysis}

Correlation between 'Dehalococcoides' and contaminant concentrations was determined by Pearson Product Moment Correlation (SigmaStat 3.5). $P$ values $>0.05$ indicate no significant relationship between the two variables. $16 \mathrm{~S}$ rDNA and tceA data were analyzed by One Way Analysis of Variance (SigmaStat 3.5). P values $<0.001$ indicate no statistically significant differences between the different samples.

\section{Results and discussion}

Chemical and geochemical characterization of the chlorinated solvent-contaminated site

The present study was carried out at a chlorinated solvent-contaminated site in Northern Italy. The site has a long history (over 20 years) of soil and groundwater contamination, most probably related to the various industrial activities which have occurred over the years, among which is the manufacturing of a range of synthetic dyes. Groundwater contamination by chlorinated hydrocarbons was most probably caused by the leakage of an underground storage tank located approximately 2-6 $\mathrm{m}$ below the ground surface (bgs), in an area which is now referred to as 'the contamination source'. Both a shallow aquifer, from 5 to $10 \mathrm{~m}$ bgs (hereafter referred to as A0) and a deeper aquifer (from 15 to $50 \mathrm{~m}$ bgs), hereafter referred to as A1, are now severely contaminated. The shallow aquifer is primarily contaminated by 1,1,2,2tetrachloroethane, whereas the deeper aquifer is primarily (and more heavily, up to $30,000 \mu \mathrm{g} \mathrm{L}^{-1}$ ) contaminated by trichloroethene (TCE) (Table 1). Figure 1 shows the spatial distribution of TeCA and TCE in the deeper aquifer based on the chemical analyses carried out on groundwater samples obtained from several monitoring wells available at the site. Most of the sampled wells (10 out of 13) were in A1 and were located downgradient to the contamination source; notably, well PE (located in A0) was in the proximity of the contamination source (Fig. 1).

Groundwater analyses also revealed the presence of lower concentrations of perchloroethylene (PCE), cis-1,2-dichloroethylene (cis-DCE), and 1,1,2-TCA (TCA) (Table 1). 1,1-DCE, trans-DCE, VC, ethene and ethane, which are also known to be TeCA and TCE dechlorination intermediates, were not detected in any groundwater sample. Interestingly, while the presence of cis-DCE and TCA suggests the occurrence of intrinsic dechlorinating activity at the site (these compounds are formed from the microbially catalyzed hydrogenolysis of TCE and TeCA, respectively), their low concentration levels suggest that the biodegradation process was most probably limited by the availability of a suitable electron donor. This is also consistent with the finding that groundwater contamination has been detected for an extremely long time (over 20 years). The measured chemical oxygen demand (COD) of groundwater samples was typically below the analytical detection limit (i.e. $<5 \mathrm{mg} \mathrm{L}^{-1}$ ) (data not shown), further supporting the hypothesis of lacking organic substrates. Additionally, both aquifers contained relatively high concentrations of nitrate (from $16 \mathrm{mg} \mathrm{L}^{-1}$ to $36 \mathrm{mg} \mathrm{L}^{-1}$ ) and sulfate (from $37 \mathrm{mg} \mathrm{L}^{-1}$ to $265 \mathrm{mg} \mathrm{L}^{-1}$ ), whereas dissolved oxygen was typically below $1 \mathrm{mg} / \mathrm{L}$ (Table 1). The resulting redox potential (ORP) of groundwater samples was in the range $150-320 \mathrm{mV}$ (shallow aquifer) and 180-310 mV (deeper aquifer). These ORP values are characteristic of ecosystems whereby nitrate reduction is the dominant terminal electron-accepting process (TEAP) [34]. It is also worth noting, that while the concentration of chlorinated solvents decreased substantially along the groundwater flow path, most probably due to a combination of factors such as dilution, dispersion, volatilization, (bio)degradation, the concentration of nitrate or sulfate appeared to be more homogeneous within each aquifer (Table 1), thereby 


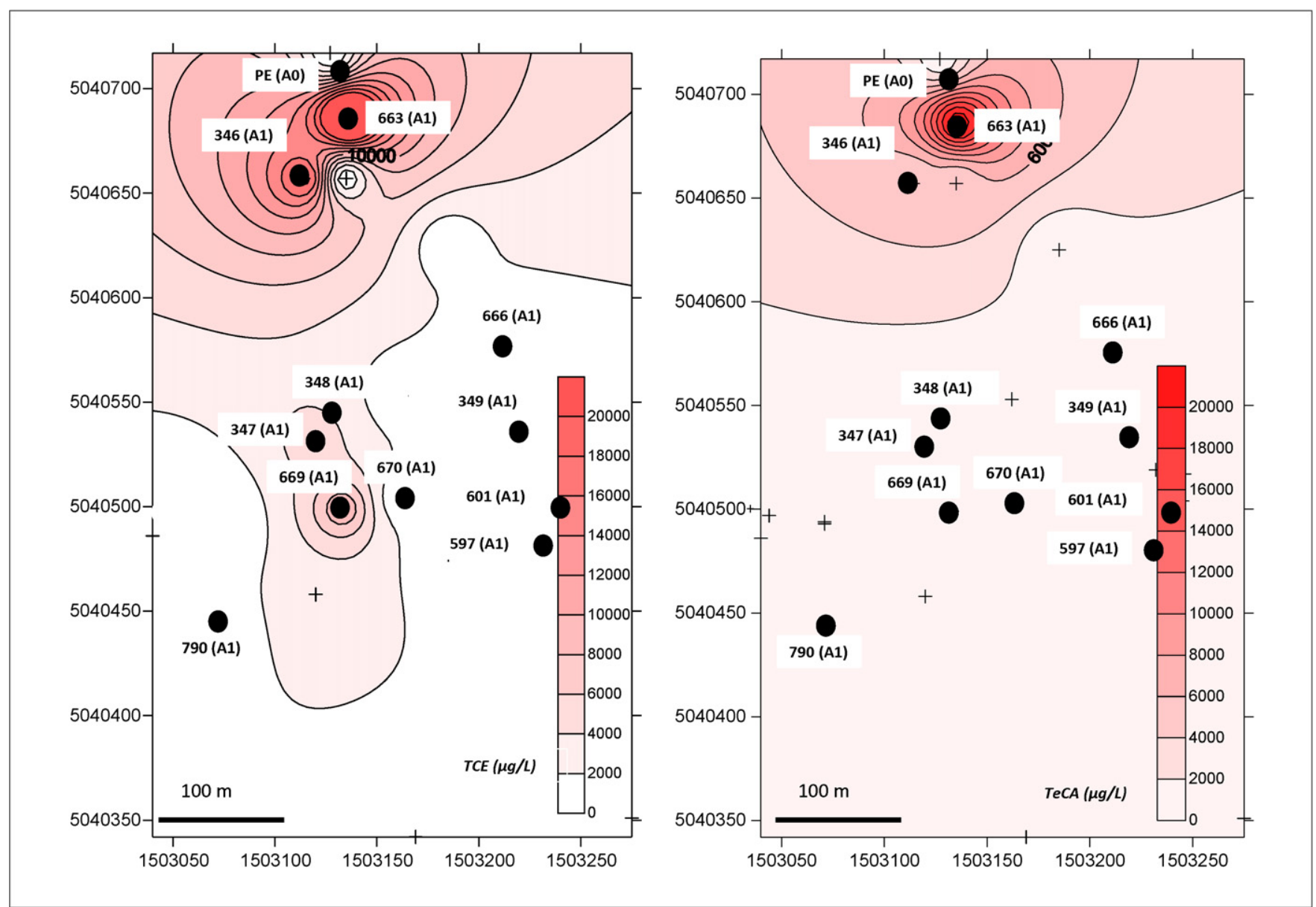

FIGURE 1

Deep aquifer (A1) monitoring wells distribution and TeCA and TCE concentration across the wells. Shallow wells (A0) 345 and 347 are located nearby wells 346 and 348, respectively. Shallow PE well is located within the contamination source nearby well 663 whereas deep well 601 is in the proximity of well 597.

suggesting that these compounds originated from different sources, than the chlorinated solvents. In all measured samples, the total (dissolved) Fe concentration was below $1 \mathrm{mg} \mathrm{L}^{-1}$ (data not shown). Groundwater $\mathrm{pH}$ was slightly acidic, ranging from 5.2 to 6.9 , whereas the electrical conductivity was in the range 0.4 $1.3 \mathrm{mS} \mathrm{cm}^{-1}$. It is worth noting that in previous studies, attempts were made to characterize the reductive dechlorination potential of the site [28,32]. To this aim, a microcosm study had been conducted with soil and groundwater sampled in the shallow aquifer, near well PE. Also in that case, the groundwater contained substantial amounts of nitrate and sulfate. All live microcosms, supplemented with fermentable substrates resulted in the stimulation of microbial dechlorinating activity by autochthonous populations. Notably, after approximately 350 days of incubation, the microcosms amended with yeast extract as electron donor displayed a nearly complete dechlorination of chlorinated contaminants to harmless ethene, pointing to a direct involvement of 'Dehalococcoides' spp. in the dechlorination process. FISH analyses had confirmed the occurrence of this microorganism in active, ethene-producing microcosms [37]. While the above described microcosm study was highly informative (e.g. degradation rates and pathways were assessed, as well as the contribution of competitive metabolisms to electron donor consumption), it was also extremely time- and labor-intensive (e.g. over 350 days were needed to observe complete dechlorination; hundreds of chemical analyses were required), and allowed screening the reductive dechlorination potential of only a single aquifer sample and accordingly did not provide any information regarding the spatial distribution of the reductive dechlorination activity at the site.

As discussed in the following paragraphs, here an alternative approach, based on the application of a suite of molecular techniques, was applied to overcome intrinsic limitations of microcosm studies, and possibly retrieve additional (and/or complementary) information on the reductive dechlorination potential of the site.

\section{Biomonitoring of contaminant key-degrading bacteria by CARD- FISH}

The abundance and activity of chlorinated solvents key-degrading bacteria here were assessed at field scale through the combined application of in situ-hybridization (CARD-FISH) and quantitative PCR-based methods (qPCR and RT-qPCR). Initially, CARD-FISH was employed for estimating cell abundances at different phylogenetic levels (from domain to genus level) with particular attention to bacteria known to be involved in the partial (i.e. Dehalobacter spp., Desulfitobacterium spp., Sulfurospirillum spp., Desulfuromonas spp.) or complete ('Dehalococcoides' spp.) dechlorination of chlorinated solvents. 

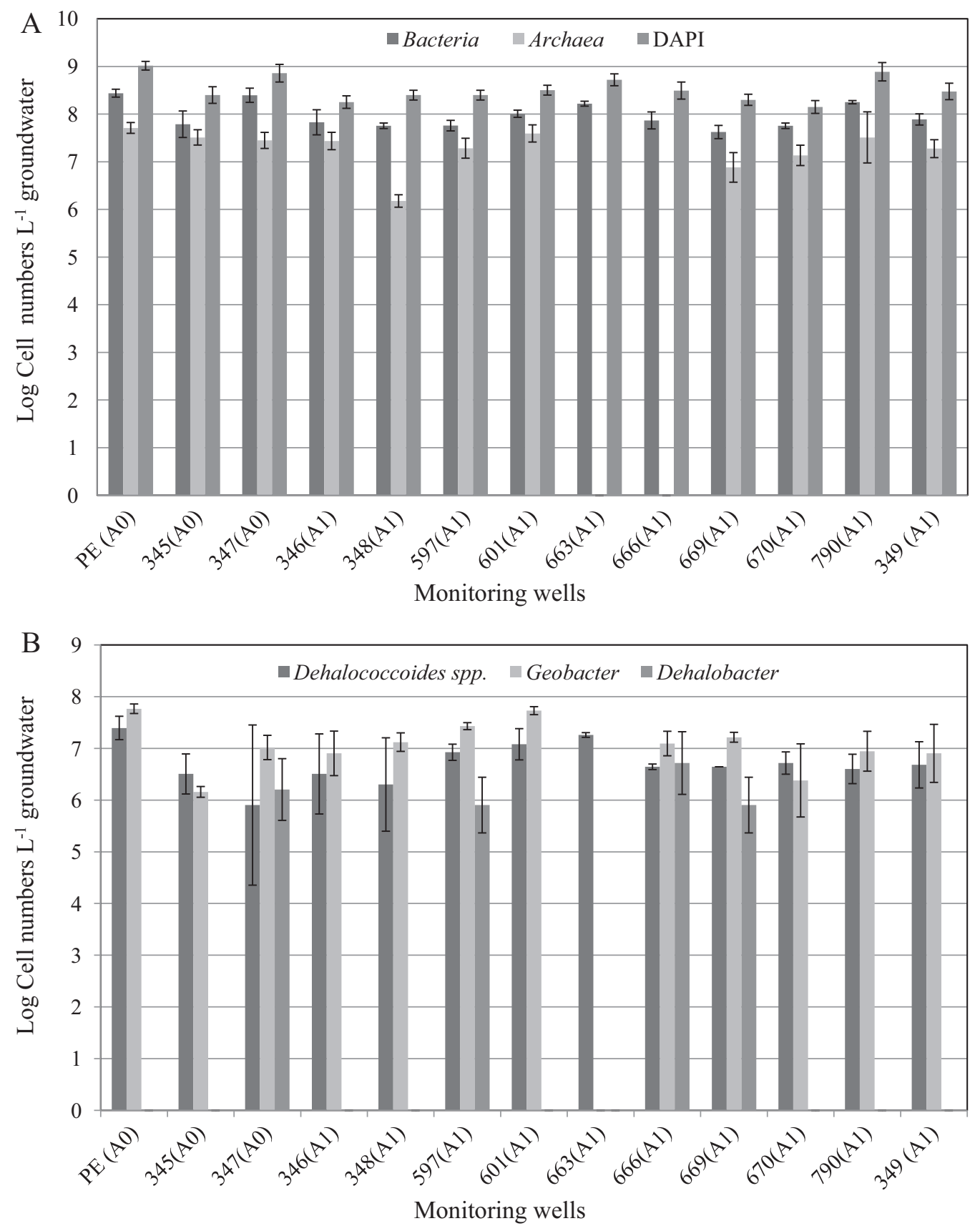

\section{FIGURE 2}

CARD-FISH quantification of total cells (DAPI), Bacteria and Archaea (a). Cells abundance of microorganisms known to be involved in anaerobic reductive dechlorination (b). Data are reported as cells $\mathrm{L}^{-1}$ of groundwater.

As a result, CARD-FISH allowed to quantify specifically targeted whole cells per volume of groundwater (cells $\mathrm{L}^{-1}$ of groundwater) and to additionally estimate the fraction out of the total microbial community (i.e. expressed as percentage out of the total DAPI stained cells or out of total Bacteria). Bacteria quantified by CARDFISH ranged between $4.2 \times 10^{7} \pm 5.8 \times 10^{6}$ and $2.7 \times 10^{8} \pm$ $2.3 \times 10^{7}$ cells L $^{-1}$ (corresponding to $7.6 \pm 1$ to $8.4 \pm 0.7 \mathrm{log}$ cells $\mathrm{L}^{-1}$ in Fig. $2 \mathrm{a}$ ), and represented almost $21 \%$ of the total cells with values up to $40 \%$ (Eub338mix/DAPI).
In spite of the relatively high ORP, Archaea were found to be abundant in groundwater samples at concentrations ranging between $1.5 \times 10^{6} \pm 1.9 \times 10^{5}$ and $5.1 \times 10^{7} \pm 5.8 \times 10^{6}$ cell numbers $\mathrm{L}^{-1}$ (corresponding to $6.1 \pm 0.8$ and $7.7 \pm 0.8 \mathrm{log}$ cells $\mathrm{L}^{-1}$ in Fig. 2a) representing thereby potential competitors of dechlorinating bacteria. Among all investigated dechlorinating bacteria ('Dehalococcoides' spp., Dehalobacter spp., Desulfitobacterium spp., Desulfuromonas spp. and Sulfurospirillum spp.) only 'Dehalococcoides' spp. and Dehalobacter spp. were detected (Fig. 2b). 
'Dehalococcoides' spp. was detected in all monitoring wells with values of cell numbers $\mathrm{L}^{-1}$ ranging from $8.1 \times 10^{5} \pm 1.2 \times 10^{5}$ to $2.5 \times 10^{7} \pm 5.6 \times 10^{6} \quad$ (corresponding to $5.9 \pm 0.9$ and $7.4 \pm 1.6 \log$ cells $\mathrm{L}^{-1}$ in Fig. $2 \mathrm{~b}$ ). Members of this genus represented $\leq 15 \%$ of the total bacterial population (\%Dhe12259c,t/ Eub338mix), with min and max relative values of $0.7 \%$ and $15 \%$ Dhe1259c,t/Eub338mix, respectively. 'Dehalococcoides' spp. was more abundant in wells located nearby the contamination source both in shallow (well PE) and in the deeper (well 663) aquifers whereas in the area downgradient to the contamination source 'Dehalococcoides' cell densities were found to be nearly homogenously distributed (Fig. 2b). 'Dehalococcoides' spp. was always detected in the contaminated groundwater samples even if, as expected, no significant correlation between 'Dehalococcoides' density and contaminant concentration was found ( $P$ values $>0.05$ for all contaminants detected in the aquifer). The lack of this correlation was also previously observed [20] and suggested to be due to the several factors which can affect, in addition to contaminant biotransformation processes, the concentration of chlorinated solvents at field scale (i.e. the dissolution or desorption of the chlorinated solvents from the source material).

Among the dechlorinating microorganisms which were able to partially reduce PCE or TCE to cis-DCE, Dehalobacter spp. was marginally detected (only in four samples) with cell numbers comparable to those obtained for 'Dehalococcoides' spp. $\left(<1.6 \times 10^{6}\right.$ cells $\left.\mathrm{L}^{-1}\right)$. In addition, a FISH probe for Geobacter spp. (GEO432 probe), previously developed on the basis of cloned $16 \mathrm{~S}$ rRNA genes in a microcosm study set up with aquifer material originating from the same site being investigated in the present study [24] was also applied for the screening of groundwater samples. Geobacter spp. detected by GEO432 probe ranged between
$1.5 \times 10^{6} \pm 1.5 \times 10^{5}$ and $5.9 \times 10^{7} \pm 4.1 \times 10^{6}$ cells $\mathrm{L}^{-1}$ (corresponding to $6.2 \pm 0.6$ and $7.8 \pm 0.5 \log$ cells $\mathrm{L}^{-1}$ in Fig. $2 \mathrm{~b}$ ) representing, in most of the samples, $>10 \%$ of the total Bacteria, up to $52 \%$. Differently from 'Dehalococcoides' spp., Geobacter spp. cannot be unequivocally linked to a single metabolic feature as members of this genus are well-known iron reducing microorganisms and only Geobacter lovleyi, strain SZ, was shown to be capable to reduce highly chlorinated solvents to cis-DCE [38,39]. However, GEO432 probe specifically binds a narrow group of 16S RNA gene sequences including the known Fe(III) reducer Geobacter chapellei and does not detect Geobacter lovleyi, strain SZ. As a result, the high cell densities of Geobacter spp. retrieved in contaminated groundwater reasonably were due to iron reducing microbial activities occurring at Rho site. Dissimilatory Fe(III) reduction turned out as the main metabolism in competition with dechlorination for electrondonor utilization during a field test conducted in a confined area at the contamination source of Rho site [40].

In previous studies, dechlorinating bacteria other than 'Dehalococcoides' spp. were described within Chloroflexi phylum. They include the DF-1/o-17 group and 1,1,2,2-TeCA dechlorinating strains BL-DC-8 and BL-DC-9 [41]. However, the application of a CARD-FISH probe specific for Chloroflexi phylum (GNSB941 probe) showed that most of Chloroflexi were 'Dehalococcoides' spp. (>90\% DHE1259c,t/GNSB941) in all screened wells. The sole exception was well 347 where 'Dehalococcoides' spp. represented only $\sim 7 \%$ of Chloroflexi. It is worth noting that, differently from the others wells, in previous sampling campaigns neither chlorinated solvents contamination was retrieved in well 347 nor 'Dehalococcoides' spp. was detected by either CARD-FISH and qPCR. This may therefore explain the low concentration of 'Dehalococcoides' spp. observed in this well. Several additional

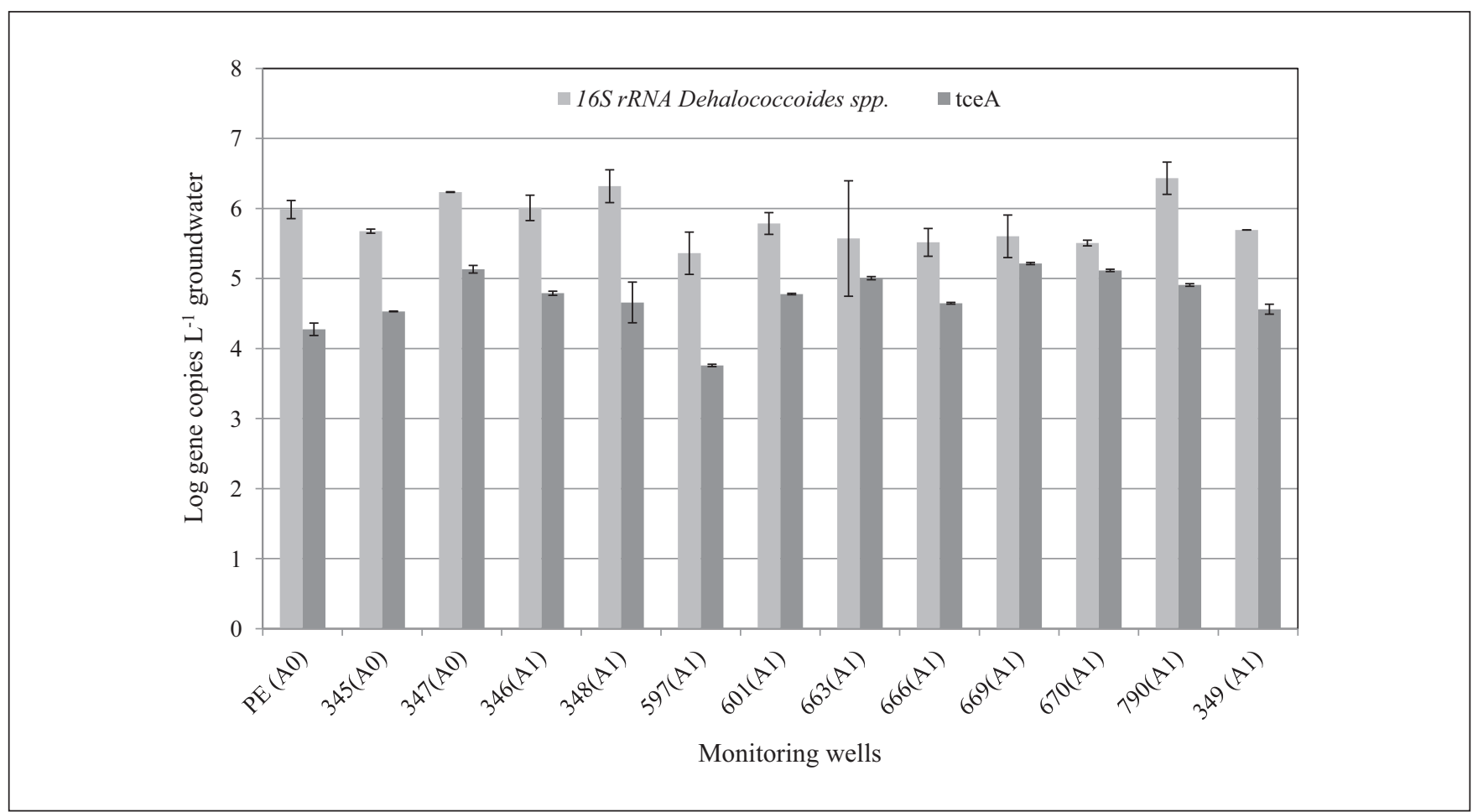

FIGURE 3

Gene copies of 'Dehalococcoides' spp. 16S rRNA and tceA in groundwater samples. Data are reported as log gene copies $\mathrm{L}^{-1}$ groundwater. 
wells available in the area (even distant from those reported in Fig. 1) were sampled to obtain an uncontaminated groundwater to be used as negative control. Surprisingly, in all of them we could detect the presence (although in some cases at low concentrations) of chlorinated solvents probably due to the presence in the area of multiple contamination sources, with some of them not even yet identified. Additional groundwater samples were taken from other chlorinated solvent contaminated sites in Italy, where we could identify wells not containing the contaminants. The results of molecular analyses (either CARDFISH or qPCR) did not reveal the occurrence of 'Dehalococcoides' spp. in all analyzed 'uncontaminated control' samples (data not shown).
Discrimination among 'Dehalococcoides' strains with different dechlorination capabilities by $q P C R$

CARD-FISH showed that 'Dehalococcoides' spp. was the main dechlorinator in the contaminated aquifer. However, this finding required a further evaluation of the dechlorination potential existing in the site. It is well known that different 'Dehalococcoides' strains, characterized by a high degree of $16 \mathrm{~S}$ rRNA gene similarity ( $>98 \%$ identity), have indeed marked differences in terms of dehalogenating capabilities [2-4,6,10]. As mentioned earlier, only 'Dehalococcoides' strains 195 and FL2 have tceA gene for proteins catalysing the metabolic dechlorination of TCE to cis-DCE and VC and for the cometabolic production of ethene. As shown in Fig. 3, the discrimination among 'Dehalococcoides' strains with different
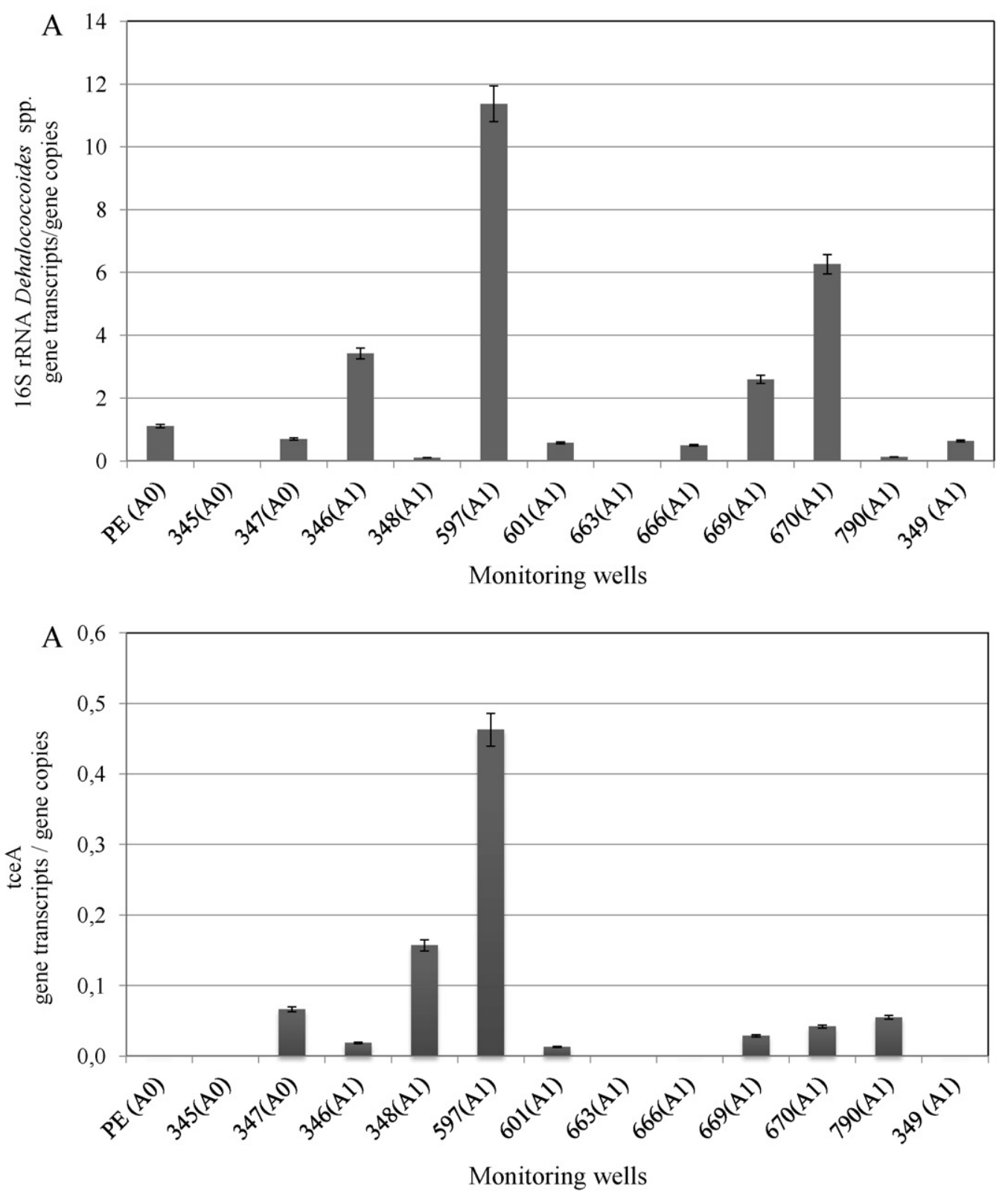
dechlorination capabilities can be done by qPCR targeting, in addition to $16 \mathrm{~S}$ rRNA gene, RDase coding genes specifically involved in different steps of the reductive dechlorination process.

As shown in Fig. 3, qPCR confirmed the widespread occurrence of 'Dehalococcoides' spp. ascertained by CARD-FISH. A direct quantitative comparison of data obtained with the two adopted methods (CARD-FISH and qPCR) is immediate for 'Dehalococcoides' spp., because $16 \mathrm{~S}$ rRNA gene is present as single copy per cell [42]. 16S rRNA gene was detected in all analyzed samples at concentration significantly different between wells $(P<0.001)$ and ranging from $2.29 \times 10^{5} \pm 1.1 \times 10^{4}$ to $2.70 \times 10^{6} \pm 1.3 \times 10^{5}$ copies $\mathrm{L}^{-1}$ (corresponding to $5.36 \pm 0.2$ to $6.43 \pm 0.3 \log$ gene copies $\mathrm{L}^{-1}$ in Fig. 3), about one order of magnitude lower than cell densities obtained by CARD-FISH. This finding is most probably due to known limitations in DNA extraction from environmental samples, which can introduce biases in nucleic acids quantification (DNA or RNA) [43].

As a consequence, also the different 'Dehalococcoides' abundances obtained by CARD-FISH in the contamination source and in the area located downgradient were not highlighted by qPCR. This finding supports the use of in situ detection methods especially when reliable estimates of biomarkers cell numbers are required for biomonitoring purposes.

Further, the abundance of functional genes tce $A, b v c A$ and $v c r A$ was analyzed by qPCR and among these, only tce $A$ gene was retrieved in all analyzed samples and ranged from $5.73 \times 10^{3} \pm 2.8 \times 10^{2}$ to $1.64 \times 10^{5} \pm 8.1 \times 10^{3}$ gene copies $\mathrm{L}^{-1}$ (corresponding to $3.75 \pm 0.1$ to $5.21 \pm 0.2 \log$ gene copies $\mathrm{L}^{-1}$ in Fig. $3 ; P<0.01)$. No $b v c A$ and $v c r A$ genes were found, according with the lack of by-products of the dechlorination process such as cis-DCE (present only at low amount) or VC and with the negligible presence of known dechlorinating bacteria able to perform a partial dechlorination (i.e. only Dehalobacter spp. was found in some of the screened wells). These results revealed that 'Dehalococcoides' cells indigenous to the site were mainly affiliated to strains carrying tceA gene, known to be capable to completely dechlorinate TCE beyond cis-DCE (strains 195 and FL2).

\section{Estimation of dechlorination activity by $R T-q P C R$}

As is known, the presence or absence of reductive dehalogenase genes may be not adequate for evaluating the metabolic activity of strains identified via functional genes. Gene expression analysis, estimated as the abundance of gene transcripts out of the gene copies, may serve as physiological biomarker of dechlorination process occurring at field scale [44]. In this regard, RT-qPCR was applied to quantify 'Dehalococcoides' spp. 16S rRNA and tceA gene expression, which were found in almost all samples with transcripts number per gene ranging from $0.1 \pm 0.004$ to $11.37 \pm 0.05$ (Fig. $4 \mathrm{a}$ ) and $0.013 \pm 0.0006$ to $0.46 \pm 0.002$, respectively (Fig. $4 \mathrm{~b}$ ) In line with the low dechlorination activity occurring at the site, 'Dehalococcoides' 16S rRNA and tceA gene expression level were several fold lower than those commonly reported for actively dechlorinating 'Dehalococcoides' cultures [19].
As shown in Fig. 4, no transcripts were detected in some monitoring wells. However, it is well known that gene expression data can be strongly affected by RNA loss during the extraction procedure, which can cause the underestimation of gene transcripts [7]. In our study the estimated RNA recovery during the extraction was about $40 \%$, in line with data reported for enrichment cultures (which ranged between 30\% and 40\%) [7].

It is worth noting that only very few RDase expression studies were previously performed at field scale and mainly conducted on bioaugmented or biostimulated sites [19]. This lack of information does not allow us to easily compare our results with literature data. Similarly, most of the RDase expression experiments were indeed mainly conducted on laboratory scale dechlorinating enrichments with tceA expression levels, as expected, higher than those estimated in this study [45]. Lower tce $A$ expression $\left(10^{-1}\right.$ and $10^{1}$ tceA transcripts per gene copies) was instead reported for culture enrichment experiments conducted at lower temperatures $\left(<20^{\circ} \mathrm{C}\right)$, under conditions closer to those typically found in groundwater [45].

RDase expression analysis showed, even though probably underestimating the extent of the process, the occurrence of RD activities at the site. This information along with the estimation of native dechlorinating bacteria cell number is valuable for the early evaluation of the remedial potential of the site where no remedial actions have been applied.

\section{Conclusions}

The study showed the effectiveness of the combined use of in situ detection and PCR-based methods to estimate cell densities and activity of key-dechlorinating bacteria, either 'Dehalococcoides' spp. or other halorespiring bacteria with limited dechlorinating capabilities, naturally occurring in chlorinated solvents contaminated sites. CARD-FISH was found to be more sensitive than qPCR for the quantification of 'Dehalococcoides' spp. cell numbers in the aquifer whereas qPCR provided the key information about the metabolic potentialities of 'Dehalococcoides' spp. by the estimation of RDases and their expression level. Moreover, as CARD-FISH can be also applied for in situ gene detection, a further improvement of this approach for detecting RDase instead of 16S rRNA genes may allow the in situ discrimination of 'Dehalococcoides' strains with different metabolic traits and enhance the application potentialities of biomonitoring activities.

\section{Acknowledgements}

The work has been carried out in the framework of EU ModelProbe Project (grant agreement no. 213161). Prof. Ruth Richardson is acknowledged for valuable suggestions and support in RT-qPCR analysis by hosting BM at her laboratory within Short Term Mobility Fellowship funded by National Research Council, Italy.

\section{Appendix A. Supplementary data}

Supplementary data associated with this article can be found, in the online version, at http://dx.doi.org/10.1016/j.nbt.2012.07.006. 


\section{References}

1 Smidt, H. and de Vos, W.M. (2004) Anaerobic microbial dehalogenation. Annu. Rev. Microbiol. 58, 43-73

2 He, J.Z. et al. (2003) Complete detoxification of vinyl chloride by anaerobic enrichment culture and identification of the reductively dechlorinating population as "Dehalococcoides" species. Appl. Environ. Microbiol. 69, 996-1003

3 Maymo-Gatell, X. et al. (1997) Isolation of a bacterium that reductively dechlorinates tetrachloroethene to ethene. Science 276, 1568-1571

4 Muller, J.A. et al. (2004) Molecular identification of the catabolic vinyl chloride reductase from "Dehalococcoides" sp. strain VS and its environmental distribution. Appl. Environ. Microbiol. 70, 4880-4888

5 Sung, Y.K. et al. (2006) Quantitative PCR confirms purity of strain GT, a novel trichloroethene (TCE)-to-ethene-respiring "Dehalococcoides" isolate. Appl. Environ. Microbiol. 72, 1980-1987

6 Adrian, L. et al. (2010) Bacterial dehalorespiration with chlorinated benzenes. Nature 408, 580-583

7 Johnson, D.R. et al. (2005) An internal reference technique for accurately quantifying specific mRNAs by real-time PCR with application to the tceA reductive dehalogenase gene. Appl. Environ. Microbiol. 71, 3866-3871

8 Bach, H.J. et al. (2002) Enumeration of total bacteria and bacteria with genes for proteolytic activity in pure cultures and in environmental samples by quantitative PCR mediated amplification. J. Microbiol. Methods 49, 235-245

9 Brunk, C.J. et al. (2002) Analysis of specific bacteria from environmental samples using a quantitative polymerase chain reaction. Curr. Issues Mol. Biol. 4, 13-18

$10 \mathrm{He}$, J. et al. (2005) Isolation and characterization of "Dehalococcoides" sp. strain FL2, a trichloroethene (TCE) and 1,2-dichloroethene-respiring anaerobe. Environ. Microbiol. 7, 1442-1450

11 Mackay, I.M. (2004) Real-time PCR in the microbiology laboratory. Clin. Microbiol. Infect. 10, 190-212

12 Magnuson, J.K. et al. (2002) Trichloroethene reductive dehalogenase from "Dehalococcoides" ethenogenes: sequence of $t c e A$ and substrate range characterization. Appl. Environ. Microbiol. 66, 5141-5147

13 Seshadri, R. et al. (2005) Genome sequence of the PCE-dechlorinating bacterium "Dehalococcoides" ethenogenes. Science 307, 105-108

14 Ding, C. and He, J. (2011) Molecular techniques in the biotechnological fight against halogenated compounds in anoxic environments. Microbial Biotechnol. 3, 347-367

15 Cupples, A.M. (2008) Real-time PCR quantification of "Dehalococcoides" populations: methods and applications. J. Microbiol. Methods 72, 1-11

16 Ritalahti, K.M. et al. (2006) Quantitative PCR targeting 16S rRNA and reductive dehalogenase genes simultaneously monitors multiple "Dehalococcoides" strains. Appl. Environ. Microbiol. 72, 2765-2774

17 Bürgmann, H. et al. (2008) Detection and quantification of Dehalococcoides-related bacteria in a chlorinated ethene-contaminated aquifer undergoing natural attenuation. Biorem. J. 12, 193-209

18 Carreón-Diazconti, C. et al. (2009) Assessment of in situ reductive dechlorination using compound-specific stable isotopes, functional gene PCR, and geochemical data. Environ. Sci. Technol. 43, 4301-4307

19 Lee, P.K.H. et al. (2008) Transcripts to assess the in situ physiology of "Dehalococcoides" spp. in a trichloroethene-contaminated groundwater site. Appl. Environ. Microbiol. 74, 2728-2739

20 Lu, X. et al. (2006) Relationship between "Dehalococcoides" DNA in ground water and rates of reductive dechlorination at field scale. Water Res. 40, 3131-3140

21 Ritalahti, K.M. et al. (2010) Comparing on-site to off-site biomass collection for "Dehalococcoides" biomarker gene quantification to predict in situ chlorinated ethene detoxification potential. Environ. Sci. Technol. 13, 5127-5133

22 Aulenta, F. et al. (2004) Detection and quantitative estimation of "Dehalococcoides" spp. in a dechlorinating bioreactor by a combination of fluorescent in situ hybridisation (FISH) and kinetic analysis. Appl. Microbiol. Biotechnol. 64, 206-212

23 Fazi, S. et al. (2008) Improved quantification of "Dehalococcoides" species by fluorescence in situ hybridization and catalyzed reporter deposition. Syst. Appl. Microbiol. 31, 62-67

24 Rossetti, S. et al. (2008) Structure analysis and performance of a microbial community from a contaminated aquifer involved in the complete reductive dechlorination of 1,1,2,2-tetrachloroethane to ethene. Biotechnol. Bioeng. 100, 240-249

25 Watts, J.E. et al. (2005) A PCR-based specific assay reveals a population of bacteria within the Chloroflexi associated with the reductive dehalogenation of polychlorinated biphenyls. Microbiology 151, 2039-2046

26 Burns, M. and Valdivia, H. (2008) Modelling the limit of detection in real-time quantitative PCR. Res. Technol. 226, 1513-1524

27 Amann, R. and Bernhard, M.F. (2008) Single-cell identification in microbial communities by improved fluorescence in situ hybridization techniques. Nat. Rev. Microbiol. 6, 339-348

28 Aulenta, F. et al. (2005) Comparative study of methanol, butyrate, and hydrogen as electron donors for long-term dechlorination of tetrachloroethene in mixed anaerobic cultures. Biotechnol. Bioeng. 91, 743-753

29 Aulenta, F. et al. (2009) Microbial reductive dechlorination of trichloroethene to ethene with electrodes serving as electron donors without the external addition of redox mediators. Biotechnol. Bioeng. 103, 85-91

30 Aulenta, F. et al. (2010) Characterization of an electro-active biocathode capable of dechlorinating trichloroethene and cis-dichloroethene to ethane. Biosens. Bioelectron. 25, 1796-1802

31 Yang, Y.R. et al. (2005) Identification of microorganisms involved in reductive dehalogenation of chlorinated ethenes in an anaerobic microbial community. Water Res. 39, 54-66

32 Aulenta, F. et al. (2006) Anaerobic bioremediation of groundwater containing a mixture of 1,1,2,2-tetrachloroethane and chloroethenes. Biodegradation 17, 193-206

33 Fagervold, S.K. et al. (2007) Microbial reductive dechlorination of Aroclor 1260 in Baltimore harbor sediment microcosms is catalyzed by three phylotypes within the phylum Chloroflexi. Appl. Environ. Microbiol. 73, 3009-3018

34 Loy, A. et al. (2007) probeBase - an online resource for rRNA-targeted oligonucleotide probes: new features 2007. Nucleic Acids Res. 35, D800D804

35 Miller, D.N. et al. (1999) Evaluation and optimization of DNA extraction and purification procedures for soil and sediment samples. Appl. Environ. Microbiol. 65, 4715-4724

36 Pernthaler, J. et al. (2001) Fluorescence in situ hybridization (FISH) with rRNAtargeted oligonucleotide probes. Methods Microbiol. 30, 207-226

37 Aulenta, F. et al. (2007) Relevance of side reactions in anaerobic reductive dechlorination microcosms amended with different electron donors. Water Res. $41,27-38$

38 Strycharz, S.M. et al. (2008) Graphite electrode as a sole electron donor for reductive dechlorination of tetrachloroethene by Geobacter lovleyi. Appl. Environ. Microbiol. 74, 5943-5947

39 Sung, Y. et al. (2006) Geobacter lovleyi sp. nov. strain SZ, a novel metal-reducing and tetrachloroethene - dechlorinating bacterium. Appl. Environ. Microbiol. 72, 27752782

40 Aulenta, F. et al. (2007) Field study in situ bioremediation of a chlorinated solvent source zone. Ind. Eng. Chem. Res. 46, 6812-6819

41 Yan, J. et al. (2009) Detection and quantification of Dehalogenimonas and "Dehalococcoides" populations via PCR-based protocols targeting 16S rRNA genes. Appl. Environ. Microbiol. 23, 7560-7564

42 Klappenbach, J.A. et al. (2001) Rrndb: the ribosomal RNA operon copy number databases. Nucleic Acids Res. 29, 181-184

43 Smits, T.H.M. et al. (2004) Development of a real-time PCR method for quantification of the three genera Dehalobacter, Dehalococcoides, and Desulfitobacterium in microbial communities. J. Microbiol. Methods 57, 369378

44 Lee, P.K.H. et al. (2006) Reductive dehalogenase gene expression as a biomarker for physiological activity of "Dehalococcoides" spp.. Appl. Environ. Microbiol. 72, 61616168

45 Johnson, D.R. et al. (2005) Transcriptional expression of the tceA gene in a Dehalococcoides-containing microbial enrichment. Appl. Environ. Microbiol. 71, 7145-7151 